\title{
Cord leptin is inversely associated with changes in weight and adiposity in infancy
}

\author{
C. ní Chaoimh ${ }^{1,2}$, D.M. Murray ${ }^{2,3}$, L.C. Kenny ${ }^{2}$, J.O’B. Hourihane ${ }^{3}$ and M. Kiely ${ }^{1,2}$ \\ ${ }^{1}$ Vitamin D Research Group, School of Food and Nutritional Sciences, University College Cork, ${ }^{2}$ The Irish Centre for \\ Fetal and Neonatal Translational Research, University College Cork and ${ }^{3}$ Department of Paediatrics and Child Health, \\ University College Cork, Republic of Ireland
}

Emerging evidence suggests that fetal exposure to low-circulating concentrations of the adipocyte-derived hormone leptin may promote a fast growth trajectory in infancy and predispose to childhood obesity ${ }^{(1,2)}$. To date, studies examining this association have used weight gain as a measure of growth, while none have used more precise measures of body composition. The aim of this study was to determine associations between umbilical cord blood leptin concentrations and changes in weight and adiposity during infancy.

Cord leptin concentrations were measured in 346 infants participating in the Cork BASELINE Birth Cohort Study. Weight was measured at birth, 2, 6, 12 and 24 months. Age-and-sex specific standard deviation scores (SDS) were generated using the UK-WHO growth reference data ${ }^{(3)}$. Fat mass and fat-free mass $[\mathrm{kg}]$ were measured by air displacement plethysmography at a mean (SD) age of $1.9(1.0)$ days and $2.1(0.3)$ months and fat mass index (FMI) and fat free mass index (FFMI) [kg/m²] were calculated. Multivariate linear regression was used to explore associations between cord leptin and (1) changes in weight SDS between specified age-intervals in the first 2 years and (2) changes in body composition between birth and 2 months. Models were adjusted for birth weight SDS, gestational age, breastfeeding status at 2 or 6 months and maternal smoking during pregnancy.

Cord leptin was associated with birth weight SDS (Spearman's $r=0.45, P<0.001)$, FMI at birth $(r=0.48, P<0.001)$ and FFMI at birth $(r=0 \cdot 13, P=0 \cdot 027)$. After adjustment for birth weight SDS, the association with FFMI disappeared $(r=-0 \cdot 09, P=0 \cdot 127)$. For the first time, we show a highly significant inverse association between cord leptin and the change in FMI between birth and 2 months (data in Table). Inverse associations between cord leptin and changes in weight SDS from birth to 2 months persisted through the 6 , 12 and 24 month assessments. However, there were no associations between cord leptin and changes in weight SDS between 2 and 6 or 6 and 12 months.

\begin{tabular}{|c|c|c|c|c|c|}
\hline Variable & $n$ & Unadjusted estimate $(95 \% \mathrm{CI})$ & $P$-value & Adjusted estimate $(95 \% \mathrm{CI})$ & $P$-value \\
\hline \multicolumn{6}{|l|}{ Change in weight SDS } \\
\hline $0-2$ months & 331 & $-0.032(-0.040,-0.025)$ & $<0.001$ & $-0.021(-0.029,-0.013)$ & $<0.001$ \\
\hline $0-6$ months & 332 & $-0.034(-0.044,-0.024)$ & $<0.001$ & $-0.016(-0.025,-0.006)$ & 0.001 \\
\hline $0-12$ months & 310 & $-0.033(-0.044,-0.022)$ & $<0.001$ & $-0.010(-0.020,0.000)$ & 0.059 \\
\hline 0-24 months & 339 & $-0.032(-0.042,-0.022)$ & $<0.001$ & $-0.011(-0.020,-0.001)$ & $0 \cdot 025$ \\
\hline $2-6$ months & 323 & $-0.003(-0.010,0.005)$ & 0.481 & $0.004(-0.004,0.012)$ & $0 \cdot 291$ \\
\hline $6-12$ months & 301 & $0.001(-0.005,0.006)$ & 0.834 & $0.004(-0.002,0.010)$ & $0 \cdot 182$ \\
\hline \multicolumn{6}{|c|}{ Change in body composition } \\
\hline FMI 0-2 months & 225 & $-0.036(-0.047,-0.026)$ & $<0.001$ & $-0.021(-0.032,-0.010)$ & $<0.001$ \\
\hline FFMI 0-2 months & 225 & $-0.015(-0.027,-0.003)$ & 0.013 & $-0.005(-0.014,0.004)$ & $0 \cdot 306$ \\
\hline
\end{tabular}

In conclusion, we have shown in a large prospective birth cohort that lower cord leptin is associated with faster weight gain from birth through to 2 years, and with greater increases in adiposity in early infancy. Low cord leptin may be a marker of an adverse intrauterine environment, leading to lower adiposity at birth and modified appetite regulation, driving faster postnatal growth. The lack of an association between cord leptin and weight gain from 2 months indicates that the early postnatal period (0-2 months) may represent a critical window whereby exposure to low leptin concentrations 'programmes' infant growth trajectory. Further longitudinal data are required to determine the long-term implications.

This work was supported by the National Children's Research Centre. Ethical approval was granted by the Clinical Research Ethics Committee of the Cork Teaching Hospitals, ref ECM 5 (9) 01/07/2008 and the study is registered with the United States National Institutes of Health Clinical Trials Registry (http://www.clinicaltrials.gov), ID: NCT01498965. The study was conducted according to the guidelines laid down in the Declaration of Helsinki.

1. Ong KK, Ahmed ML, Sherriff A et al. (1999) J Clin Endocrinol Metab 84, 1145-1148

2. Parker M, Rifas-Shiman SL, Belfort MB et al. (2011) J Pediatr 158, 227-233

3. The UK_WHO Growth Charts: Early Years. London: RCPCH, 2009. 